\title{
Factors influencing seed germination of the pastoral plant Retama raetam subsp. bovei (Fabaceae): interactive effects of fruit morphology, salinity, and osmotic stress
}

\author{
Kaouther Mechergui ${ }^{1}$, \\ Hela Mahmoudi², \\ Mohamed Larbi Khouja ${ }^{1}$, \\ Wahbi Jaouadi ${ }^{1,3^{*}}$ \\ ${ }^{1}$ Laboratory of Forest Ecology, \\ National Research Institute \\ of Rural Engineering, \\ Water, and Forestry (INRGREF), \\ University of Tunis Carthage. \\ Rue Hedi Karay B.P.Nº 10 Ariana 2080, Tunisia \\ ${ }^{2}$ Unit of Physiology and Biochemistry \\ of Plant Responses to Abiotic Constraints, \\ Faculty of Sciences of Tunis, \\ University of Tunis El Manar, \\ 2092 Tunis, Tunisia \\ ${ }^{3}$ The Sylvo-Pastoral Institute of Tabarka, \\ BP. $n^{\circ} 345$, Tabarka 8110 , \\ Jendouba University, Tunisia

The aim of this work was to study the morphology of retama seeds and the combined effects of salt and water stress on the seeds germination of four Tunisian populations of Retama raetam. Seeds were harvested from Bouhedma National Park, Meknassi, Oueslatia, and Rtiba. In this study we were interested in evaluating different parameters of germination in presence of the salinity and osmotic stress. The results revealed that this species can withstand salinity up to $15 \mathrm{~g} / \mathrm{l}$ of salt (the germination percentage of the Rtiba population reached $42 \%$ ) and it can also tolerate severe drought resulted in significant doses of $\mathrm{PEG}_{6000}$ (germination percentage reached $29 \%$ to the water potential of $-1.6 \mathrm{MPa}$ of Oueslatia population). The morphological traits of the seeds were measured to find a relationship between the size of the seeds and their germination capacity under salinity and osmotic stress. Results have shown that for the correlation between morphological traits of seeds and the germination percentage, the seed weight is a major factor in promoting germination under stress and confirmed that saline water and sprouting inhibition have impact on the small caliber of seeds.

Keywords: Retama raetam, population, morphological traits, salt stress, water stress, germination

\section{INTRODUCTION}

The genus Retama belongs to the family Fabaceae (500 genera and 1,000 species). It includes three species (Retama monosperma, Retama raetam and Retama sphaerocarpa) with a large distribution in the East Mediterranean region, North Africa, and in the Canary Islands (Mahane 2009). Retama rae-

\footnotetext{
* Corresponding author. E-mail: jaouadiwahbi@gmail.com
}

tam, locally named as "R'tm", is a desert shrub native to several countries of North Africa (Algeria, Egypt, Libya, Morocco, and Tunisia), temperate Asia (Israel, Jordan, Lebanon, Palestine, and Syria), and South-eastern Europe (Sicily in Italy) (GRIN Database, 2011). In Morocco, it is largely located in desert regions and in the Middle Atlas. Retama raetam with steno-Mediterranean and saharo-sindic distribution mainly grows in dune and desert habitats where it can withstand extreme aridity. For this reason it is often used in dune stabilization and in 
restoration of desert areas (Boulila et al., 2009). Retama reatam has a very productive vertical and horizontal root system that can reach $20 \mathrm{~m}$. The Retama species contributes to the bio-fertilisation of poor soils because of its aptitude to associate with fixing nitrogen bacteria Rhizobia (Boulila et al., 2009; Selami et al., 2014). Therefore, the genus of the Retama is included in a revegetation programme for degraded areas in semi-arid Mediterranean environments (Caravaca et al., 2003). Retama raetam is also a medicinal and aromatic plant present in humid to arid bioclimatic regions and it is a famous herbal drug in Tunisia (Saad et al., 2014). Actually, in traditional medicine, Retama raetam is used for the treatment of several diseases such as diabetes and hypertension, and its shoots are also used as an antidote against snake bites (Maghrani, 2005). Research undertaken on genus Retama showed that the aqueous extract had diuretic and hypoglycaemic effects (Maghrani, 2005). Even more, oral administration of $20 \mathrm{mg} / \mathrm{kg}$ of this aqueous extract reduced significantly the glucose levels in the blood of diabetic rats (Maghrani, 2005). Moreover, administration of Retama raetam aqueous extracts induces a decrease in the triglyceride concentrations in the plasma of normal and diabetic rats and leads to a significant decrease in weight (Maghrani, 2005). All the fractions from the Tunisian halophyte Retama raetam showed a potent antioxidant activity and some of them exhibited interesting antibacterial qualities (Saad et al., 2014). The phenolic concentration in this species is very high, especially for the ethyl acetate fraction, and is probably responsible for its important biological activities (Saad et al., 2014). The RP-HPLC data revealed a large number of phenolic acids and flavonoids in Retama raetam shoots, with coumarin and syringic acid as major phenolics. Also, Retama raetam is a source of natural antioxidants useful in medical and food industries (Saad et al., 2014). In the south-eastern region of Morocco (Tafilalet), this plant is largely recommended by traditional herbal healers for diabetes control and phytotherapy. This ethnobotanical information has been recently verified experimentally (Maghrani et al., 2003). The un- derlying mechanism of this pharmacological effect has been hypothesised as inhibition of renal glucose reabsorption (Maghrani et al., 2005). Retama raetam species has developed a particularly important root system and is adapted to arid areas. Cellulose remains the major component of the cell wall (27\% for young roots and $80 \%$ for adult roots), hemicelluloses (14.3\% for young roots and $3.6 \%$ for adult roots) and pectins (17.3\% for young roots and $4.1 \%$ for adult roots) (Bokhari et al., 2015). Previous pharmacological studies on this plant have revealed its antibacterial, antifungal, antihypertensive, anti-oxidant, antiviral, diuretic, and hypoglycemic properties, and hepatoprotective, nephroprotective and cytotoxic effects (Maghrani et al., 2005; Eddouks et al., 2007; Hayet et al., 2007, 2008; Koriem et al., 2009; Algandaby et al., 2010; Edrizi et al., 2010). Retama raetam appears to be a valuable candidate as a forage resource in Sinai (Egypt). This fodder species should be considered valuable nonconventional forage in the Mediterranean arid ecosystem (Naser et al., 2013). Although present in low quantities in branches/leaves and seeds of Retama monosper$m a$, lipids are an important fraction in terms of quality given their high unsaturated fatty acids, particularly oleic, linoleic and linolenic acids whose therapeutic virtues are highly recommended (El Hamdani, Fdil, 2015). The fact that the linoleic acid is considered to be beneficial in cancer and diabetic prevention provides some scientific basis for the traditional use of the plant as an antidiabetic, and for its pharmaceutical indication as antileukemic (El Hamdani \& Fdil, 2015). Based on this, further pharmacological investigations to screen other potential bioactivities of fatty acids of Retama monosperma may be recommended (El Hamdani, Fdil, 2015). The highest mineral content is that of $\mathrm{Al}, \mathrm{Fe}, \mathrm{Mg}$, $\mathrm{Zn}, \mathrm{Ca}, \mathrm{K}, \mathrm{Na}$, and $\mathrm{P}$. However, the composition of the plant is mainly dependent on the composition of the soil which is influenced primarily by the nature of the rocks from which the soil is derived. In Algeria, the plant grows in Sahara and Atlas regions and is used in folk medicine to reduce blood glucose and skin inflammations (Baba, 1999) The Saharan plant Retama raetam 
(Fabaceae family) is used as a locally available adsorbent for removal of copper ions from aqueous solution. The aerial parts of Retama raetam can be used as an inexpensive biosorbent and as suitable alternatives for the removal of copper ions from wastewater (Cheriti et al., 2009). Among xerophytic shrubs, Retama raetam has a potential economic importance. It plays a significant role in soil protection and stabilization against wind or water erosion and provides an important dietary source for livestock species such as camels, goats, and sheep (Laudadio, 2009). Additionally, this species represents a viable fuel source for humans (Cheriti et al., 2009). It also has medicinal and potential industrial values since its roots are used to treat diarrhoea, the leaves are used to help aching joints, back pain, and eye troubles (Said et al., 2002). Shrubs are key components in these ecosystems as they influence both biotic and abiotic conditions. Woody species may create "islands of fertility" by improving the availability of water and nutrients (Moro et al., 1997) or by protecting against direct irradiance and overheating (Moro et al., 1997; Lopez-Pintor et al., 2000). In addition, the legume species can increase soil fertility due to nitrogen-enriched litter deposition or direct release of nitrogen from roots (Dart, 1998). Recently, researchers have become interested in woody legumes due to their ecological importance (Ndiaye, Ganry, 1997; Dart, 1998; El-Shaer, 2000). Information on the nutritive value of forage could help range management in selecting suitable grazing sites to sustain animal life without inflicting vegetation damage (Arzani et al., 2004). Thanks to high palatability of Retama raetam (Laudadio, 2009), this legume may represent an important forage resource for livestock species, especially during the dry season when shortages of pasture are common in this Mediterranean region. However, very little attention has been given to the forage potential of wild leguminous species, especially to trees and shrubs (Dart; 1998). So, the ecological potentiality of Retama raetam can contribute to the reduction of fodder shortages in the arid areas of the Mediterranean ecosystem. Retama raetam has a strong potential as a forage crop with a valu- able nutritional quality for browsing animals. Moreover, this species may represent an alternative feedstuff to the conventional forage and a promising substitute fodder in the Mediterranean ecosystem. (Naser et al., 2013). Concerning seed germination, literature data (Gutterman, 1993; Izhaki, Ne'eman, 1997; Seglie et al., 2012) show that the percentage of germination in $R e$ tama is very low due to physical dormancy caused by the impermeability of seed coat to water (Kigel, 1995). Hence, studies have also been carried out on seed morphology and seed germination under abiotic factors (salt stress and osmotic stress) to get new data on the endemic taxon of Tunisia Retama raetam subsp. bovei in view of conservation and habitat restoration.

\section{MATERIALS AND METHODS}

\section{Plant material}

Seeds of Retama raetam were collected in 2014 from four natural habitats, namely, Meknassi, Bouhedma, Rtiba, and Oueslatia (Fig. 1). After the collection, the seeds were stored in a cold room. The climatic conditions prevailing in each region were considered. Before the germination tests, damaged and insect-infected seeds were discarded, and the empty ones were eliminated using the method of floating in distilled water. Seeds were separated from fruits and soaked in water for $24 \mathrm{~h}$. These seeds were sterilised with Benlate $(1 \mathrm{~g} / \mathrm{l})$ for $20 \mathrm{~min}$, then with sodium hypochlorite $50 \%$ for few minutes, and rinsed three times with distilled water.

\section{Morphological traits}

Some specific characteristics of Retama populations were chosen as descriptors. Three were qualitative traits: the length, the width, and the weight of the seeds. Thirty seeds for each Retama populations were analyzed.

\section{Salinity and drought treatments}

To determine the tolerance on the germination stage of each Retama population under salt stress and water stress, seeds were sown in a $\mathrm{NaCl}$ solution at different concentrations (0 (distilled water), $3,6,9,12$, and $15 \mathrm{~g}$ of $\mathrm{NaCl}$ 


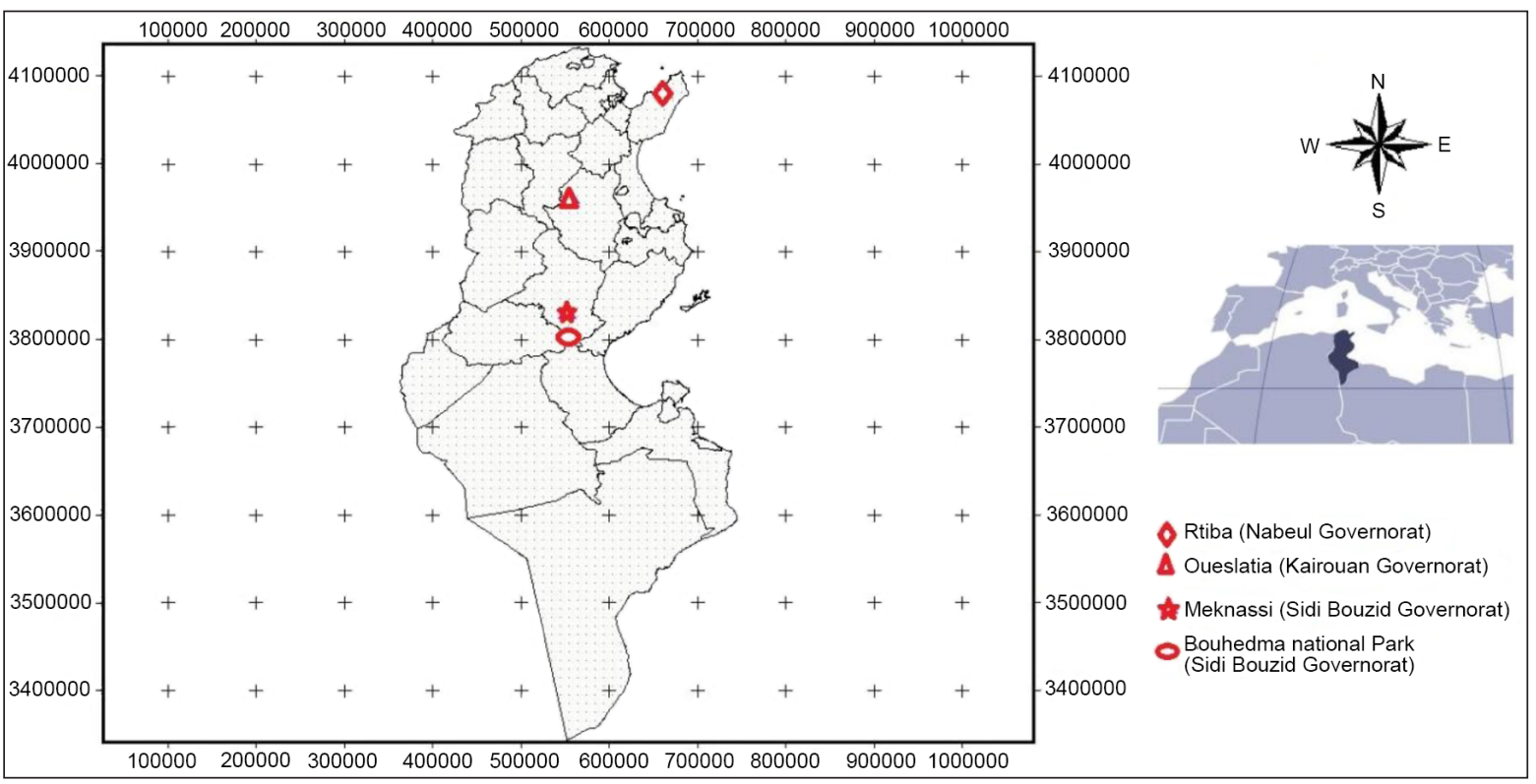

Fig. 1. Geographical location of the studied populations of Retama reatam

added to one litre of distilled water). The water stress treatments used were 0 (control), -0.03 , $-0.1,-0.7,-1$ and $-1.6 \mathrm{MPa}$ of $\mathrm{PEG}_{6000}$ added to one litre of distilled water. Seeds were placed in sterile Petri dishes with two discs of filter paper saturated with distilled water for control, and $\mathrm{NaCl}$ solutions for treatments. $\mathrm{NaCl}$ solutions were renewed every $48 \mathrm{~h}$ under sterile conditions in order to avoid salt accumulation (Rahman et al., 2008). Five replicates of 20 seeds each were used for each treatment with $10 \mathrm{ml}$ of test solution. Seeds were allowed to germinate in relative humidity of $80 \%$ at $25^{\circ} \mathrm{C}$ in complete darkness for 30 days (Maraghni et al., 2010). A seed was considered to have germinated when the emerging radicle elongated to $2 \mathrm{~mm}$ (Redondo-Gomez et al., 2007).

\section{Methods of germination expression}

The germination rate (GR) is an estimate of the viability of a population of seeds. The germination percentage was calculated using the equation below:

\section{GR = seeds germinated/total seed $\times \mathbf{1 0 0}$}

The mean time of germination (MTG) and the parameters of the germination speed were also calculated for supplementary explanations. The MTG was calculated as follows:

$$
\mathrm{MTG}=\sum n_{i} \times d_{i} / n
$$

where $n$ is the total number of germinated seeds during the germination test, $n_{i}$ is the number of germinated seeds on day $d_{i}$ and $i$ is the number of days during the germination period (between 0 and 30 days) (Yousheng \& Sziklai 1985). Germination counts were performed daily for 30 days. The germination percentage (GP \%) was evaluated daily and the final value was obtained after 30 days. The Kotowski's coefficient (CV) was calculated according to this method:

$$
\mathrm{CV}=\sum(n \times J n) / \sum n
$$

\section{Correlation between morphological traits and germination properties}

A correlation between morphological parameters and their germination rate, the average time of germination, and their velocity coefficient was determined.

\section{Statistical analysis}

Data were analysed using SPSS for Windows, version 11.5 (SPSS, 2002). A two-way analysis of variance (ANOVA) was carried out to test the effects of main factors (morphology and population/salinity and population/ drought and population) and their interaction on the rate and the final percentage of 
germination. SNK test (Student, Newmen and Keuls) was used to estimate the least significant range between the means.

\section{RESULTS}

\section{Morphological traits}

For four Retama populations, the length varied from 4.08 to $6.42 \mathrm{~mm}$, the weight varied from 5.16 to $8.77 \mathrm{~g}$, and the width varied from 1.19 to $1.93 \mathrm{~mm}$. Our results show that there is no significant variation of morphological traits of the four Retama seeds populations (Table 1 and Fig. 2). According to Table 2 and Fig. 3, population 2 has the longest seeds $(4.65 \mathrm{~mm})$ and population 1 has the maximum weight of seeds $(1.68 \mathrm{~g})$ and the maximum width of seeds $(5.98 \mathrm{~mm})$. In order to describe and gain a better understanding of variance sources among the studied Retama populations, principal component analysis (PCA) was carried out. A clear separation of the studied populations was observed and four main groups could be distinguished (Figs. 4, 5). The Euclidean distance assigned the four Retama populations into three groups (Fig. 6). The first group included population A (population 1), the second group included population B (population 2), and the third group was divided into two subgroups: the first subgroup included population $\mathrm{C}$ (population 3 ) and the second was represented by population $\mathrm{D}$ (population 4 ).

Table 1. Descriptive analysis of seed morphology of four populations of Retama raetam

\begin{tabular}{c|c|c|c|c|c|c|c|c|c|c}
\hline Variable & $\mathrm{N}$ & Minimum & Maximum & Mean & SD & $\begin{array}{c}\text { SE } \\
\text { (mean) }\end{array}$ & CV & $\begin{array}{c}\mathrm{F} \\
\text { value }\end{array}$ & $\operatorname{Pr}(>\mathrm{F})$ & $\begin{array}{c}\text { Signifi- } \\
\text { cation }\end{array}$ \\
\hline $\begin{array}{c}\text { Length of } \\
\text { seeds (mm) }\end{array}$ & 120 & 4.085 & 6.416 & 4.589 & 0.261 & 0.023 & 0.056 & 1.721 & 0.167 & $\mathrm{NS}$ \\
\hline $\begin{array}{c}\text { Weight of } \\
\text { seeds (g) }\end{array}$ & 120 & 5.165 & 8.768 & 1.650 & 0.117 & 0.010 & 0.071 & 1.998 & 0.118 & $\mathrm{NS}$ \\
\hline $\begin{array}{c}\text { Width of } \\
\text { seeds }(\mathrm{mm})\end{array}$ & 120 & 1.190 & 1.934 & 5.824 & 0.465 & 0.042 & 0.079 & 1.813 & 0.149 & $\mathrm{NS}$ \\
\hline
\end{tabular}

Significant difference from control at ${ }^{*}=P<0.05,{ }^{* *}=P<0.01,{ }^{* *}=P<0.001$. NS $=$ not significant $(P>0.05)$.

Table 2. Descriptive analysis of seed morphology of the individuals of each studied population of Retama raetam (Population 1: Rtiba; Population 2: Oueslatia; Population 3: Meknassi; Population 4: Bouhedma National Park)

\begin{tabular}{|c|c|c|c|c|c|c|}
\hline Variable & Population & mean & SD & SE (mean) & $\mathrm{CV}$ & $\mathrm{N}$ \\
\hline \multirow{4}{*}{$\begin{array}{l}\text { Length of seeds } \\
\qquad(\mathrm{mm})\end{array}$} & Population 1 (Rtiba) & 4.596 & 0.140 & 0.025 & 0.030 & 30 \\
\hline & Population 2 (Oueslatia) & 4.655 & 0.221 & 0.040 & 0.047 & 30 \\
\hline & Population 3 (Meknassi) & 4.505 & 0.412 & 0.075 & 0.091 & 30 \\
\hline & Population 4 (Bouhedma National Park) & 4.602 & 0.171 & 0.031 & 0.037 & 30 \\
\hline \multirow{4}{*}{ Weight of seeds (g) } & Population 1 (Rtiba) & 1.683 & 0.157 & 0.028 & 0.093 & 30 \\
\hline & Population 2 (Oueslatia) & 1.668 & 0.104 & 0.019 & 0.062 & 30 \\
\hline & Population 3 (Meknassi) & 1.625 & 0.088 & 0.016 & 0.054 & 30 \\
\hline & Population 4 (Bouhedma National Park) & 1.625 & 0.100 & 0.018 & 0.061 & 30 \\
\hline \multirow{4}{*}{$\begin{array}{l}\text { Width of seeds } \\
\qquad(\mathrm{mm})\end{array}$} & Population 1 (Rtiba) & 5.987 & 0.569 & 0.103 & 0.095 & 30 \\
\hline & Population 2 (Oueslatia) & 5.813 & 0.215 & 0.039 & 0.036 & 30 \\
\hline & Population 3 (Meknassi) & 5.760 & 0.666 & 0.121 & 0.115 & 30 \\
\hline & Population 4 (Bouhedma National Park) & 5.737 & 0.184 & 0.033 & 0.032 & 30 \\
\hline
\end{tabular}



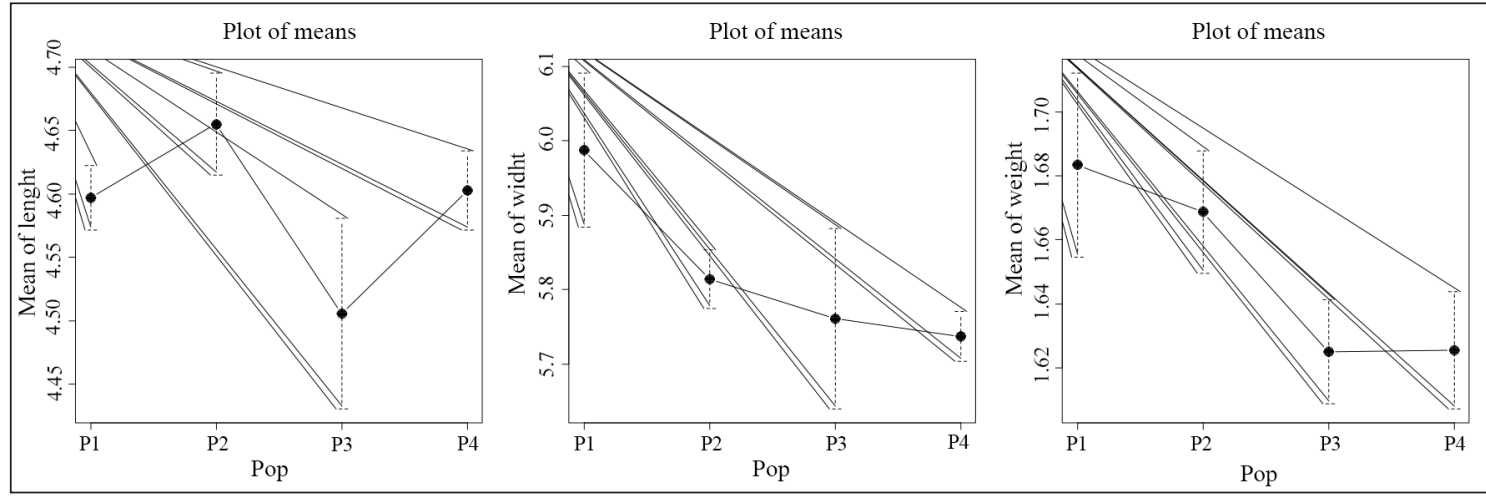

Fig. 2. Averages $( \pm S D)$ of three morphological parameters of four populations (Pop) of Retama reatam (P1: Rtiba; P2: Oueslatia; P3: Meknassi; P4: Bouhedma National Park)

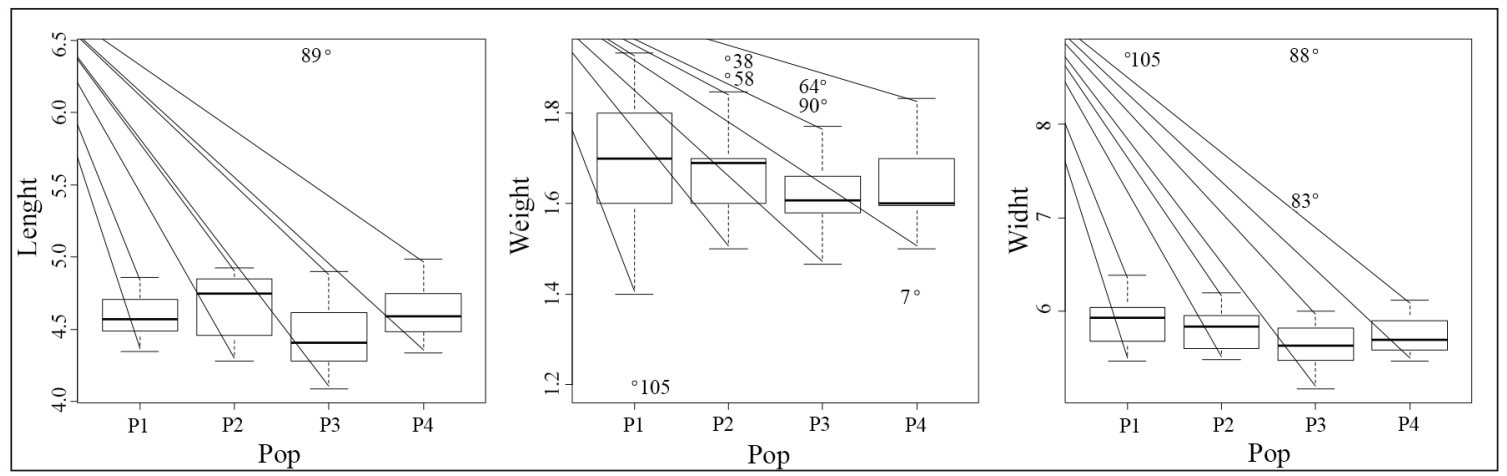

Fig. 3. Box dispersion of three morphological parameters of four populations (Pop) of Retama reatam (P1: Rtiba; P2: Oueslatia; P3: Meknassi; P4: Bouhedma National Park)

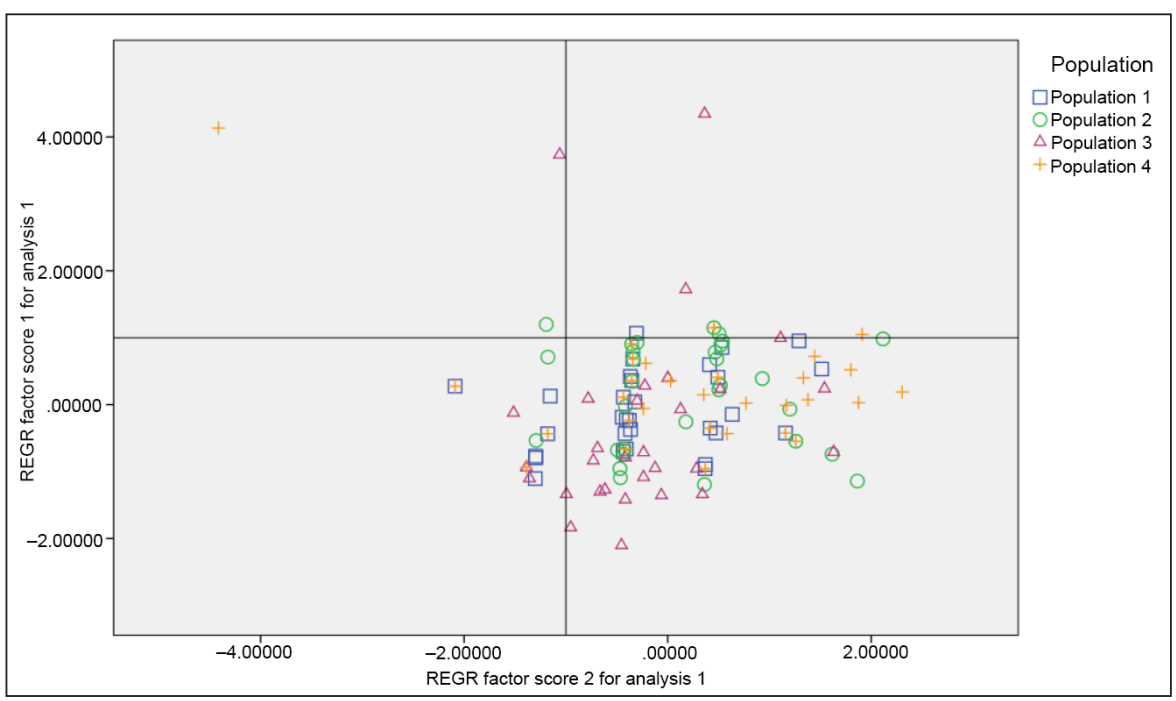

Fig. 4. Principal components analysis of seeds morphology of each studied population individuals of Retama reatam using the SPSS program (Population 1: Rtiba; Population 2: Oueslatia; Population 3: Meknassi, Population 4: Bouhedma National Park) 


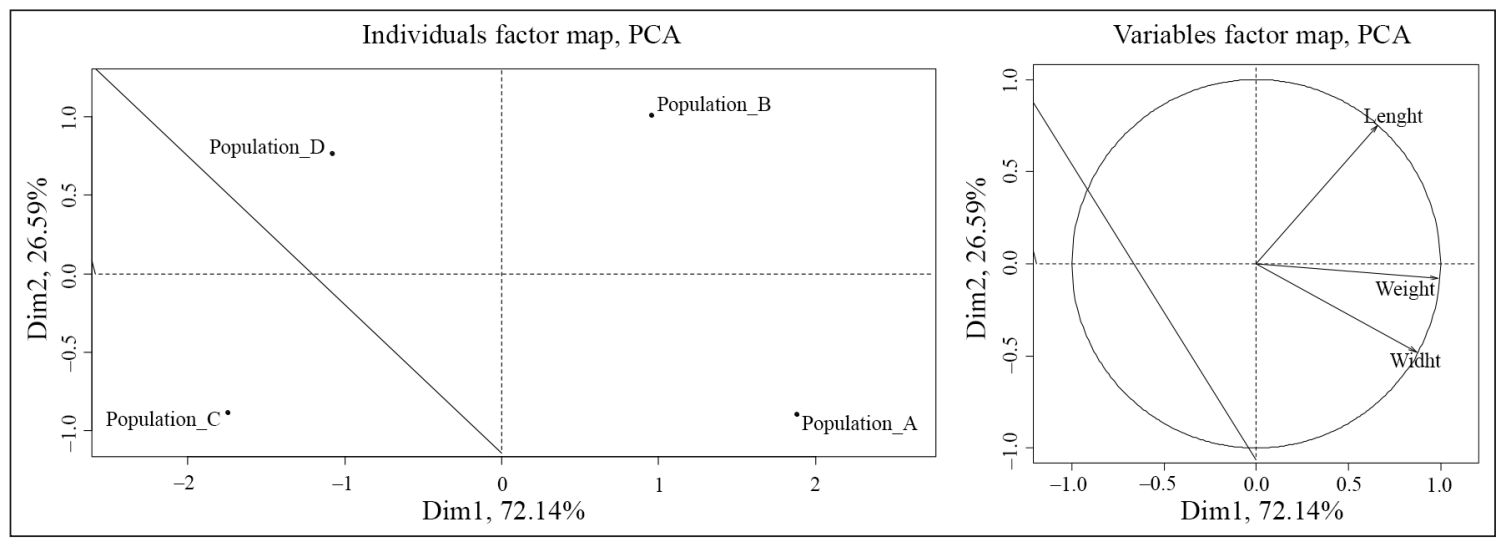

Fig. 5. Principal components analysis of Retama reatam populations using the $\mathrm{R}$ program; Individuals factor map and variables factor map (Population_A: Rtiba; Population_B: Oueslatia; Population_C: Meknassi, Population_D: Bouhedma National Park)

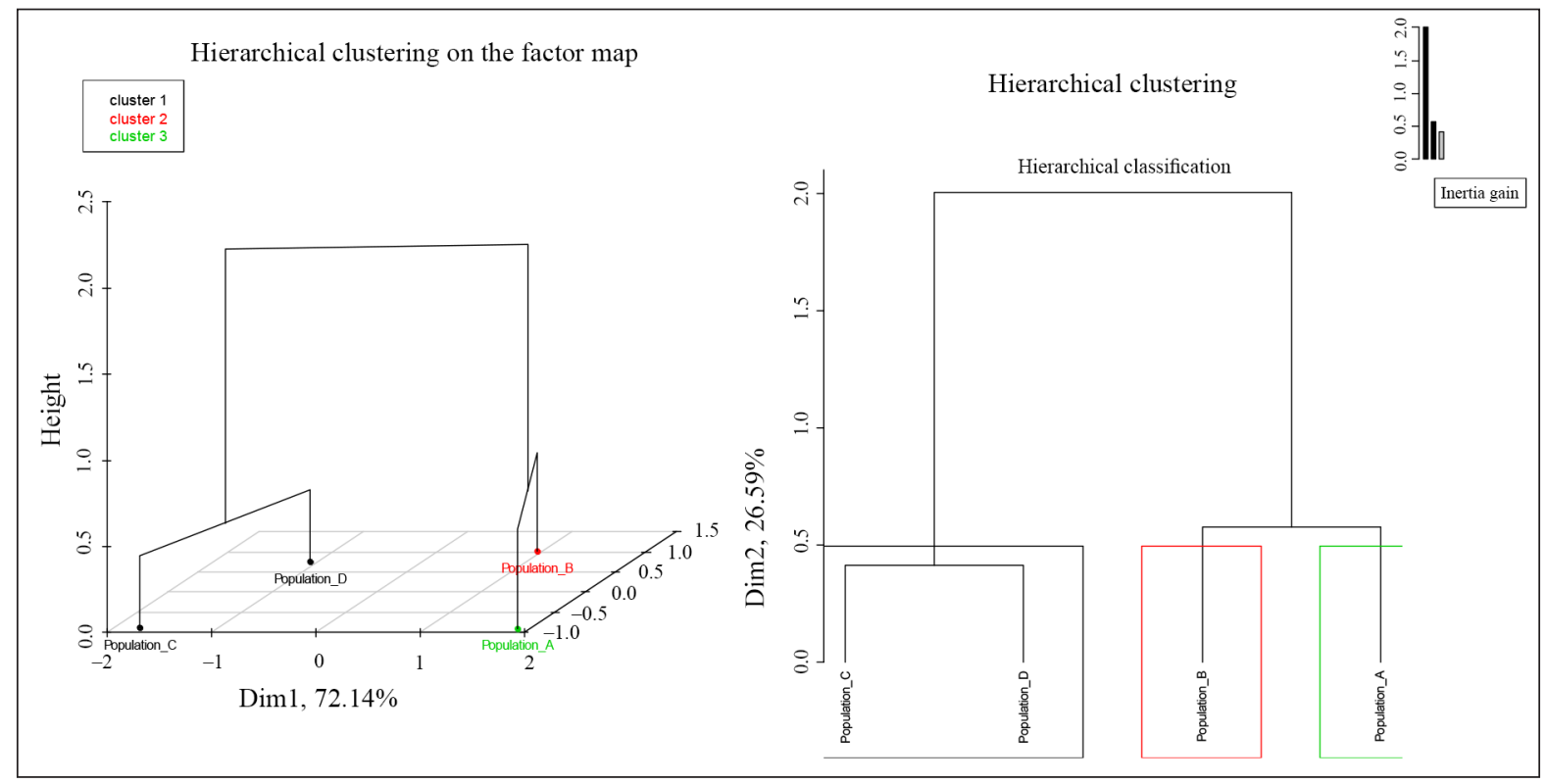

Fig. 6. Euclidean distance between the four Retama reatam population susing the R program (Population_A: Rtiba; Population_B: Oueslatia; Population_C: Meknassi, Population_D: Bouhedma National Park)

\section{Effects of salt stress on seed germination}

A two-way ANOVA indicated a significant interaction between population and salinity from Kotowski's coefficient (Table 3). The germination percentage varied significantly between the four Retama populations $(P<0.0001)$. Salinity had a significant effect on the germination percentage, Kotowski's coefficient and the mean time of germination $(P<0.0001)$. The effects of salinity constraint $(\mathrm{NaCl}$ treatment) on seed germination are reported in $\mathrm{Ta}$ - ble 4. The results of ANOVA showed that $\mathrm{NaCl}$ treatments had a significant effect on the germination percentage and mean germination time (Table 4). However, salinity significantly affected the germination percentage of Retama populations (Table 4). Germination in distilled water was the highest, it varied between $81 \%$ at $73 \%$. However, it decreased significantly with increased $\mathrm{NaCl}$ concentrations (Table 4). Population 1 is the most resistant to salinity ( $42 \%$ of germination percentage at $15 \mathrm{~g} / \mathrm{l}$ ), population 4 
Table 3. A two-way ANOVA of the effects of salinity, population, and their interaction on germination characteristics of germination of four populations of Retama reatam seeds

\begin{tabular}{|c|c|c|c|c|}
\hline Source & Dependent Variable & F-value & P-value & Signification \\
\hline \multirow{3}{*}{ Population } & Germination percentage & 20.489 & 0.000 & $* * *$ \\
\hline & Kotowski's coefficient & 0.906 & 0.441 & NS \\
\hline & Mean time of germination & 2.706 & 0.050 & NS \\
\hline \multirow{3}{*}{ Concentration } & Germination percentage & 30.127 & 0.000 & $* * *$ \\
\hline & Kotowski's coefficient & 35.144 & 0.000 & $* * *$ \\
\hline & Mean time of germination & 26.403 & 0.000 & $* * *$ \\
\hline \multirow{3}{*}{ Population ${ }^{\star}$ Concentration } & Germination percentage & 0.911 & 0.554 & NS \\
\hline & Kotowski's coefficient & 1.832 & 0.041 & $* *$ \\
\hline & Mean time of germination & 0.969 & 0.493 & NS \\
\hline
\end{tabular}

Significant difference from control at ${ }^{\star}=P<0.05,{ }^{* *}=P<0.01$, ${ }^{* *}=P<0.001$ by SNK test. NS $=$ not significant $(P>0.05)$.

Table 4. Characteristic of germination of four populations of Retama reatam after their transfer from $0,3,6,9,12$, and $15 \mathrm{~g} / 1$ of salinity at $25^{\circ} \mathrm{C}$. Data represented means $\pm \mathrm{SD}$. Different letters indicate significant differences between treatments (Salinity) at $\boldsymbol{P}<0.05$ according to the SNK test. (Population 1: Rtiba; Population 2: Oueslatia; Population 3: Meknassi; Population 4: Bouhedma National Park)

\begin{tabular}{|c|c|c|c|c|c|c|c|}
\hline \multirow[b]{2}{*}{ Population } & \multirow{2}{*}{$\begin{array}{l}\text { Characteris- } \\
\text { tic of germi- } \\
\text { nation }\end{array}$} & \multicolumn{6}{|c|}{ Salinity (g/l) } \\
\hline & & 0 & 3 & 6 & 9 & 12 & 15 \\
\hline \multirow{3}{*}{$\begin{array}{l}\text { Popula- } \\
\text { tion } 1 \\
\text { (Rtiba) }\end{array}$} & $\begin{array}{c}\text { Germination } \\
\text { percentage }\end{array}$ & $81 \pm 12.45^{c}$ & $71 \pm 14.47^{b c}$ & $64 \pm 8.21^{\mathrm{abc}}$ & $60 \pm 15.41^{\mathrm{abc}}$ & $53 \pm 15.24^{\mathrm{ab}}$ & $42 \pm 20.18^{a}$ \\
\hline & $\begin{array}{l}\text { Kotowski's } \\
\text { coefficient }\end{array}$ & $13.41 \pm 3.13^{\mathrm{d}}$ & $10.15 \pm 0.77^{c}$ & $8.86 \pm 1.54^{\mathrm{bc}}$ & $5.85 \pm 1.23^{\mathrm{ab}}$ & $7.24 \pm 0.55^{\mathrm{ab}}$ & $5.94 \pm 0.34^{\mathrm{a}}$ \\
\hline & $\begin{array}{l}\text { Mean time of } \\
\text { germination }\end{array}$ & $7.92 \pm 2.53^{\mathrm{a}}$ & $9.89 \pm 0.76^{\mathrm{ab}}$ & $11.58 \pm 2.15^{\mathrm{bc}}$ & $14.95 \pm 2.62^{\mathrm{de}}$ & $13.87 \pm 1.07^{\mathrm{cd}}$ & $16.87 \pm 0.99^{\mathrm{e}}$ \\
\hline \multirow{3}{*}{$\begin{array}{c}\text { Popula- } \\
\text { tion } 2 \\
\text { (Oueslatia) }\end{array}$} & $\begin{array}{l}\text { Germination } \\
\text { percentage }\end{array}$ & $86 \pm 9.61^{b}$ & $50 \pm 16.95^{a}$ & $42 \pm 23.61^{\mathrm{a}}$ & $39 \pm 25.43^{\mathrm{a}}$ & $29 \pm 20.43^{a}$ & $28 \pm 27.74^{\mathrm{a}}$ \\
\hline & $\begin{array}{l}\text { Kotowski's } \\
\text { coefficient }\end{array}$ & $14.66 \pm 4.09^{\mathrm{b}}$ & $8.41 \pm 2.07^{\mathrm{a}}$ & $7.57 \pm 0.60^{\mathrm{a}}$ & $6.44 \pm 1.33^{\mathrm{a}}$ & $7.03 \pm 2.08^{\mathrm{a}}$ & $5.18 \pm 1.21^{\mathrm{a}}$ \\
\hline & $\begin{array}{l}\text { Mean time of } \\
\text { germination }\end{array}$ & $7.25 \pm 1.96^{\mathrm{a}}$ & $12.48 \pm 3.09^{b}$ & $13.26 \pm 1.06^{\mathrm{b}}$ & $16.02 \pm 3.03^{b c}$ & $15.46 \pm 5.38^{\mathrm{bc}}$ & $20.19 \pm 4.92^{\mathrm{c}}$ \\
\hline \multirow{3}{*}{$\begin{array}{c}\text { Popula- } \\
\text { tion } 3 \\
\text { (Meknassi) }\end{array}$} & $\begin{array}{l}\text { Germination } \\
\text { percentage }\end{array}$ & $73 \pm 15.24^{\mathrm{b}}$ & $47 \pm 25.64^{a}$ & $45 \pm 17.32^{\mathrm{a}}$ & $30 \pm 10.60^{\mathrm{a}}$ & $27 \pm 18.23^{\mathrm{a}}$ & $19 \pm 11.93^{\mathrm{a}}$ \\
\hline & $\begin{array}{l}\text { Kotowski's } \\
\text { coefficient }\end{array}$ & $18.32 \pm 5.35^{\mathrm{b}}$ & $8.97 \pm 3.23^{\mathrm{a}}$ & $8.32 \pm 2.27^{\mathrm{a}}$ & $6.06 \pm 1.57^{\mathrm{a}}$ & $4.70 \pm 0.84^{\mathrm{a}}$ & $4.45 \pm 0.57^{\mathrm{a}}$ \\
\hline & $\begin{array}{l}\text { Mean time of } \\
\text { germination }\end{array}$ & $5.84 \pm 1.56^{\mathrm{a}}$ & $12.88 \pm 6.31^{b}$ & $12.58 \pm 2.66^{\mathrm{b}}$ & $17.24 \pm 3.59^{\mathrm{bc}}$ & $21.78 \pm 3.54^{\mathrm{c}}$ & $22.76 \pm 2.94^{c}$ \\
\hline \multirow{3}{*}{$\begin{array}{c}\text { Popula- } \\
\text { tion } 4 \\
\text { (Bouhedma } \\
\text { National } \\
\text { Park) }\end{array}$} & $\begin{array}{c}\text { Germination } \\
\text { percentage }\end{array}$ & $75 \pm 5.00^{\mathrm{d}}$ & $47 \pm 4.47^{\mathrm{c}}$ & $23 \pm 11.51^{b}$ & $17 \pm 10.36^{\mathrm{ab}}$ & $10 \pm 7.07^{a}$ & $08 \pm 4.47^{\mathrm{a}}$ \\
\hline & $\begin{array}{l}\text { Kotowski’s } \\
\text { coefficient } \\
\end{array}$ & $13.93 \pm 1.88^{\mathrm{b}}$ & $09.39 \pm 3.67^{\mathrm{ab}}$ & $13.16 \pm 5.58^{b}$ & $8.24 \pm 5.12^{\mathrm{ab}}$ & $5.69 \pm 1.70^{\mathrm{a}}$ & $5.39 \pm 1.87^{\mathrm{a}}$ \\
\hline & $\begin{array}{l}\text { Mean time of } \\
\text { germination }\end{array}$ & $7.28 \pm 0.98^{\mathrm{a}}$ & $12.71 \pm 7.16^{\mathrm{ab}}$ & $9.14 \pm 4.83^{\mathrm{ab}}$ & $15.90 \pm 8.47^{\mathrm{ab}}$ & $18.75 \pm 5.16^{\mathrm{b}}$ & $20.26 \pm 6.62^{b}$ \\
\hline
\end{tabular}


is the most sensitive to salinity ( $8 \%$ of the germination percentage at $15 \mathrm{~g} / \mathrm{l}$ ). There was a strong negative relationship between germination and salinity. The index of the germination velocity calculated using Kotowski's coefficient showed that the rate decreased with an increase in salt concentration (Table 4). The germination speed expressed by the mean time of germination decreased with the decrease in $\mathrm{NaCl}$ potentials. The germination was significantly reduced by high $\mathrm{NaCl}$ levels and there were no great differences in the final germination percentage between 3 and $9 \mathrm{~g} / \mathrm{l}$, so the germination percentage was reduced with increasing $\mathrm{NaCl}$ to levels above $15 \mathrm{~g} / \mathrm{l}$ (SNK test).

\section{Correlation between morphological traits and germination properties}

Table 5 shows that there is a correlation between the germination percentage and Kotowski's coefficient, the germination percentage and the mean time of germination, and between Kotowski's coefficient and the mean time of germination. As for the correlation between morphological traits and germination properties, we noticed a correlation between the weight of seeds and the germination percentage, and between the weight and the length of seeds.

\section{Effects of the osmotic potential on seed ger- mination}

A two-way ANOVA of the germination percentage, Kotowski's coefficient and the mean time of germination indicated a significant effect of the osmotic potential concentrations and high interaction between the osmotic potential and population (Table 6). The germination characteristics varied significantly between Retama populations. The effects of water stress (PEG treatment) on seed germination are reported in Table 7. The osmotic potential significantly affected the percentage of germination of $R e$ tama populations (Table 7). The germination percentage reached $94 \%$ to $99 \%$ in control seeds (0 MPa). However, it decreased significantly with an increase of the osmotic potential (Table 7). Population 2 was the most resistant to the osmotic potential $(29 \%$ of the germination percentage at $-1.6 \mathrm{MPa}$ ), population 1 was the most sensitive one (10\% of the germination percentage). The mean time of germination increased with increasing osmotic potential concentra-

Table 5. Correlation between characteristic of germination and morphology of seeds of four populations of Retama reatam after their transfer of salinity

\begin{tabular}{|c|c|c|c|c|c|c|c|}
\hline Variable & Correlation & $\begin{array}{c}\text { Germination } \\
\text { percentage }\end{array}$ & $\begin{array}{l}\text { Kotowski's } \\
\text { coefficient }\end{array}$ & $\begin{array}{l}\text { Mean time of } \\
\text { germination }\end{array}$ & $\begin{array}{l}\text { Length } \\
\text { of seeds }\end{array}$ & $\begin{array}{c}\text { Width } \\
\text { of seeds }\end{array}$ & $\begin{array}{l}\text { Weight } \\
\text { of seeds }\end{array}$ \\
\hline \multirow{2}{*}{$\begin{array}{l}\text { Germination } \\
\text { percentage }\end{array}$} & Pearson Correlation & 1 & $0.528^{* *}$ & $-0.508^{* *}$ & -0.212 & -0.064 & $0.376^{*}$ \\
\hline & Sig. (2-tailed) & & 0.003 & 0.004 & 0.261 & 0.737 & 0.041 \\
\hline \multirow{2}{*}{$\begin{array}{l}\text { Kotowski's } \\
\text { coefficient }\end{array}$} & Pearson Correlation & $0.528^{* *}$ & 1 & $-0.953^{* *}$ & -0.198 & -0.233 & -0.276 \\
\hline & Sig. (2-tailed) & 0.003 & & 0.000 & 0.295 & 0.216 & 0.139 \\
\hline \multirow{2}{*}{$\begin{array}{l}\text { Mean time of } \\
\text { germination }\end{array}$} & Pearson Correlation & $-0.508^{* *}$ & $-0.953^{* *}$ & 1 & 0.160 & 0.217 & 0.269 \\
\hline & Sig. (2-tailed) & 0.004 & 0.000 & & 0.399 & 0.250 & 0.151 \\
\hline \multirow{2}{*}{$\begin{array}{l}\text { Lenght of } \\
\text { seeds }\end{array}$} & Pearson Correlation & -0.212 & -0.198 & 0.160 & 1 & 0.180 & $0.822^{* *}$ \\
\hline & Sig. (2-tailed) & 0.261 & 0.295 & 0.399 & & 0.340 & 0.000 \\
\hline \multirow{2}{*}{ Widht of seeds } & Pearson Correlation & -0.064 & -0.233 & 0.217 & 0.180 & 1 & 0.225 \\
\hline & Sig. (2-tailed) & 0.737 & 0.216 & 0.250 & 0.340 & & 0.233 \\
\hline \multirow{2}{*}{$\begin{array}{l}\text { Weight of } \\
\text { seeds }\end{array}$} & Pearson Correlation & $-0.376^{*}$ & -0.276 & 0.269 & $0.822^{* *}$ & 0.225 & 1 \\
\hline & Sig. (2-tailed) & 0.041 & 0.139 & 0.151 & 0.000 & 0.233 & \\
\hline
\end{tabular}

${ }^{* *}$ Correlation is significant at the 0.01 level (2-tailed)

* Correlation is significant at the 0.05 level (2-tailed) 
Table 6. A two-way ANOVA of the effects of Osmotic potential, population, and their interaction on germination characteristics of four population of Retama raetam

\begin{tabular}{|c|c|c|c|c|}
\hline Source & Dependent Variable & $F$-value & $P$-value & Signification \\
\hline \multirow{3}{*}{ Population } & Germination percentage & 6.951 & 0.000 & $* * *$ \\
\hline & Kotowski's coefficient & 7.398 & 0.000 & $* * *$ \\
\hline & Mean time of germination & 15.855 & 0.000 & $* * *$ \\
\hline \multirow{3}{*}{ Concentration } & Germination percentage & 116.391 & 0.000 & $* * *$ \\
\hline & Kotowski's coefficient & 13.313 & 0.000 & $* * *$ \\
\hline & Mean time of germination & 9.111 & 0.000 & $* * *$ \\
\hline \multirow{3}{*}{ Population ${ }^{\star}$ concentration } & Germination percentage & 0.841 & 0.631 & NS \\
\hline & Kotowski's coefficient & 4.767 & 0.000 & $* * *$ \\
\hline & Mean time of germination & 3.915 & 0.000 & $* * *$ \\
\hline
\end{tabular}

Significant difference from control at ${ }^{*}=P<0.05,{ }^{* *}=P<0.01,{ }^{* *}=P<0.001$ by SNK test. NS $=$ not significant $(P>0.05)$.

Table 7. Characteristic of germination of four populations of Retama reatam after their transfer from $0,-0.03,-0.1,-0.7,-1$ and $-1.6 \mathrm{MPa}$ of osmotic potential at $25^{\circ} \mathrm{C}$. Data represent means $\pm \mathrm{SD}$. Different letters indicate significant differences between treatments (Osmotic potential) at $\mathbf{P}<0.05$ according to the SNK test. (Population 1: Rtiba; Population 2: Oueslatia; Population 3: Meknassi; Population 4: Bouhedma National Park)

\begin{tabular}{|c|c|c|c|c|c|c|c|}
\hline \multirow{2}{*}{$\begin{array}{l}\text { Popula- } \\
\text { tion }\end{array}$} & \multirow{2}{*}{$\begin{array}{l}\text { Characteristic } \\
\text { of germination }\end{array}$} & \multicolumn{6}{|c|}{ Osmotic potential (MPa) } \\
\hline & & 0 & -0.03 & -0.1 & -0.7 & -1 & -1.6 \\
\hline \multirow{3}{*}{$\begin{array}{l}\text { Popula- } \\
\text { tion } 1 \\
\text { (Rtiba) }\end{array}$} & $\begin{array}{c}\text { Germination } \\
\text { percentage }\end{array}$ & $96 \pm 4.18^{c}$ & $46 \pm 17.10^{\mathrm{b}}$ & $27 \pm 12.04^{\mathrm{a}}$ & $24 \pm 8.21^{\mathrm{a}}$ & $14 \pm 4,18^{\mathrm{a}}$ & $10 \pm 6.18^{\mathrm{a}}$ \\
\hline & $\begin{array}{l}\text { Kotowski’s } \\
\text { coefficient }\end{array}$ & $9.10 \pm 1.62^{\mathrm{b}}$ & $7.27 \pm 1.23^{\mathrm{b}}$ & $8.21 \pm 8.48^{b}$ & $7.6 \pm 1.48^{\mathrm{b}}$ & $7.81 \pm 2.29^{b}$ & $14.35 \pm 6.26^{c}$ \\
\hline & $\begin{array}{l}\text { Mean time of } \\
\text { germination }\end{array}$ & $11.29 \pm 2.2^{\mathrm{a}}$ & $14.05 \pm 2.29^{\mathrm{a}}$ & $13.30 \pm 4.18^{\mathrm{a}}$ & $13.66 \pm 3.37^{\mathrm{a}}$ & $14.05 \pm 5.50^{\mathrm{a}}$ & $17.85 \pm 2.66^{\mathrm{a}}$ \\
\hline \multirow{3}{*}{$\begin{array}{l}\text { Popula- } \\
\text { tion } 2 \\
\text { (Ouesla- } \\
\text { tia) }\end{array}$} & $\begin{array}{l}\text { Germination } \\
\text { percentage }\end{array}$ & $99 \pm 2.23^{b}$ & $52 \pm 17.88^{\mathrm{a}}$ & $50 \pm 19.68 a$ & $33 \pm 7.58^{a}$ & $32 \pm 17.17^{a}$ & $29 \pm 15.57^{a}$ \\
\hline & $\begin{array}{l}\text { Kotowski's } \\
\text { coefficient }\end{array}$ & $20.27 \pm 4.33^{b}$ & $8.58 \pm 1.28^{\mathrm{b}}$ & $6.92 \pm 1.01^{\mathrm{b}}$ & $6.30 \pm 1.40^{\mathrm{b}}$ & $6.33 \pm 1.44^{\mathrm{b}}$ & $5.94 \pm 0.33^{c}$ \\
\hline & $\begin{array}{l}\text { Mean time of } \\
\text { germination }\end{array}$ & $5.10 \pm 1.01^{\mathrm{a}}$ & $11.86 \pm 1.72^{\mathrm{b}}$ & $14.69 \pm 2.20^{\mathrm{bc}}$ & $16.41 \pm 3.23^{c}$ & $16.35 \pm 3.19^{c}$ & $16.87 \pm 1.03^{c}$ \\
\hline \multirow{3}{*}{$\begin{array}{c}\text { Popula- } \\
\text { tion } 3 \\
\text { (Me- } \\
\text { knassi) }\end{array}$} & $\begin{array}{l}\text { Germination } \\
\text { percentage }\end{array}$ & $96 \pm 2.23^{b}$ & $41 \pm 15.57^{\mathrm{a}}$ & $33 \pm 15.24 a$ & $33 \pm 12.55^{\mathrm{a}}$ & $30 \pm 07.07^{\mathrm{a}}$ & $21 \pm 04.18^{\mathrm{a}}$ \\
\hline & $\begin{array}{l}\text { Kotowski's } \\
\text { coefficient }\end{array}$ & $17.08 \pm 5.65^{\mathrm{b}}$ & $11.15 \pm 2.63^{\mathrm{ab}}$ & $11.15 \pm 2.56^{\mathrm{ab}}$ & $9.47 \pm 1.15^{\mathrm{b}}$ & $11.52 \pm 4.77^{\mathrm{ab}}$ & $8.69 \pm 3.78^{b}$ \\
\hline & $\begin{array}{l}\text { Mean time of } \\
\text { germination }\end{array}$ & $6.38 \pm 2.02^{a}$ & $9.42 \pm 2.44^{\mathrm{ab}}$ & $9.50 \pm 2.71^{\mathrm{ab}}$ & $10.77 \pm 1.77^{\mathrm{ab}}$ & $09.75 \pm 3.47^{\mathrm{ab}}$ & $12.90 \pm 4.20^{\mathrm{b}}$ \\
\hline $\begin{array}{l}\text { Popula- } \\
\text { tion } 4\end{array}$ & $\begin{array}{l}\text { Germination } \\
\text { percentage }\end{array}$ & $94 \pm 4.18^{\mathrm{a}}$ & $37 \pm 09^{b}$ & $30 \pm 16.95^{b}$ & $27 \pm 8.36^{b}$ & $25 \pm 11.72^{b}$ & $22 \pm 05.70^{\mathrm{b}}$ \\
\hline $\begin{array}{l}\text { (Bouhed- } \\
\text { ma }\end{array}$ & $\begin{array}{l}\text { Kotowski's } \\
\text { coefficient }\end{array}$ & $15.23 \pm 2.54^{\mathrm{a}}$ & $10.50 \pm 2.61^{\mathrm{a}}$ & $9.98 \pm 1.91^{\mathrm{a}}$ & $12.17 \pm 4.80^{\mathrm{a}}$ & $13.60 \pm 1.92^{\mathrm{a}}$ & $9.64 \pm 3.29^{\mathrm{a}}$ \\
\hline $\begin{array}{l}\text { National } \\
\text { Park) }\end{array}$ & $\begin{array}{l}\text { Mean time of } \\
\text { germination }\end{array}$ & $6.72 \pm 1.22^{\mathrm{a}}$ & $10.00 \pm 2.46^{\mathrm{a}}$ & $10.33 \pm 2.12^{\mathrm{a}}$ & $09.35 \pm 3.85^{\mathrm{a}}$ & $07.46 \pm 1.04^{\mathrm{a}}$ & $11.22 \pm 3.21^{\mathrm{a}}$ \\
\hline
\end{tabular}


tions (Table 7). There was a strong negative relationship between the germination percentage and the osmotic potential. The index of the germination velocity calculated using Kotowski's coefficient showed that the rate decreased with an increase in osmotic potential concentrations.

\section{Correlation between morphological traits and germination properties}

Table 8 shows that there was a correlation between the germination percentage and Kotowski's coefficient, the germination percentage and the mean time of germination, and between Kotowski's coefficient and the mean time of germination. As regards the correlation between morphological traits and germination properties, we noticed a correlation between the weight of seeds and the germination percentage.

\section{DISCUSSION}

\section{Morphological traits}

In this study, we used morphological traits to assess the variation among four Tunisian Retama populations. For all traits analysed, significant differences between the studied populations were found. In fact, a substantial variation and important heterogeneity between these populations were observed for qualitative traits. Furthermore, this study showed that the qualitative morphological characters differed significantly among the four Retama populations and that they were influenced by environmental factors. Principal component analysis for qualitative morphological characteristics of these populations showed that a reduced number of descriptors could be used efficiently to discriminate among them. Diallo et al., (2008), in a study of the change in biometric characteristics of seeds and seedlings of nine provenances of Tamarindus indica L. (Caesalpinioideae) proposed that differences between variables were highly significant. In the seed study, Diallo et al., (2008) obtained a group linked to Indian and East African provenances, another linked to West African origins, and a middle group from Thailand. The dendrogram obtained from phenotypic similarities (seed and seedling variables) shows a structure by geographical area. The phenotypic

Table 8. Correlation between characteristics of germination and morphology of seeds of four populations of Retama after their transfer of osmotic potential

\begin{tabular}{|c|c|c|c|c|c|c|c|}
\hline Variable & Correlation & $\begin{array}{l}\text { Germina- } \\
\text { tion per- } \\
\text { centage }\end{array}$ & $\begin{array}{l}\text { Kotowski's } \\
\text { coefficient }\end{array}$ & $\begin{array}{l}\text { Mean time } \\
\text { of germi- } \\
\text { nation }\end{array}$ & $\begin{array}{l}\text { Length } \\
\text { of seeds }\end{array}$ & $\begin{array}{l}\text { Width } \\
\text { of seeds }\end{array}$ & $\begin{array}{l}\text { Weight } \\
\text { of seeds }\end{array}$ \\
\hline \multirow[t]{2}{*}{$\begin{array}{c}\text { Germination } \\
\text { percentage }\end{array}$} & Pearson Correlation & 1 & $0.391^{\star *}$ & $-0.350^{\star *}$ & 0.072 & -0.023 & $0.186^{\star}$ \\
\hline & Sig. (2-tailed) & & 0.000 & 0.000 & 0.432 & 0.803 & 0.042 \\
\hline \multirow{2}{*}{$\begin{array}{l}\text { Kotowski's } \\
\text { coefficient }\end{array}$} & Pearson Correlation & $0.391^{\star *}$ & 1 & $-0.895^{\star \star}$ & 0.043 & 0.119 & 0.018 \\
\hline & Sig. (2-tailed) & 0.000 & & 0.000 & 0.644 & 0.197 & 0.847 \\
\hline \multirow{2}{*}{$\begin{array}{l}\text { Mean time of } \\
\text { germination }\end{array}$} & Pearson Correlation & $-0.350^{* *}$ & $-0.895^{\star *}$ & 1 & -0.006 & -0.139 & -0.053 \\
\hline & Sig. (2-tailed) & 0.000 & 0.000 & & 0.948 & 0.131 & 0.566 \\
\hline \multirow{2}{*}{$\begin{array}{c}\text { Length of } \\
\text { seeds }\end{array}$} & Pearson Correlation & 0.072 & 0.043 & -0.006 & 1 & $0.283^{\star *}$ & 0.008 \\
\hline & Sig. (2-tailed) & 0.432 & 0.644 & 0.948 & & 0.002 & 0.931 \\
\hline \multirow{2}{*}{$\begin{array}{l}\text { Width of } \\
\text { seeds }\end{array}$} & Pearson Correlation & -0.023 & 0.119 & -0.139 & $0.283^{* *}$ & 1 & -0.053 \\
\hline & Sig. (2-tailed) & 0.803 & 0.197 & 0.131 & 0.002 & & 0.564 \\
\hline \multirow{2}{*}{$\begin{array}{l}\text { Weight of } \\
\text { seeds }\end{array}$} & Pearson Correlation & $0.186^{*}$ & 0.018 & -0.053 & 0.008 & -0.053 & 1 \\
\hline & Sig. (2-tailed) & 0.042 & 0.847 & 0.566 & 0.931 & 0.564 & \\
\hline
\end{tabular}

** Correlation is significant at the 0.01 level (2-tailed)

* Correlation is significant at the 0.05 level (2-tailed) 
inter-provenance variability of seedlings and seeds combined with the correlations between variables could be used to select early provenances according to the objectives defined for the selection (Diallo et al., 2008). Kouyaté, Van Damme (2002), showed that there was variability between Detarium microcarpum populations, which related to the characteristics measured on the fruit, the seed and the leaf. Three shapes of mature fruit and one shape of seed were identified. Results obtained do not make it possible to confirm the existence of different varieties within the species Detarium microcarpum (Kouyaté, Van Damme, 2002). Kouyaté et al. (2011) showed that there was significant variability which was observed according to the provenance of the baobab tree (Adansonia digitata L.) in Mali. The discriminating morphological descriptors for the baobab in Mali are the fruit length and width, the fruit peduncle length, the leaf length and width, and the number of lobes. Assogbadjo et al., 2005, showed that the morphometric variables made it possible not only to make a rather precise typology of various forms of baobab tree capsules (Adansonia digitata L.) in Benin, but also to estimate their production starting from predictive models. The variability of the baobab capsule production in various climatic zones could be a useful parameter for genetic improvement of the species answering the needs and the means of the rural populations. The seed number and the total seed mass were the best fruit characteristics for the prediction of the tree type (Cooper et al., 2003). Seed weights of Metrosideros excelsa differed between trees within categories (filled and unfilled seeds). Fertile seeds had a lower length/ width ratio than unfilled seeds. Measurements of length and width varied less than their allometric ratio (Gabriele et al., 2002). Components analyses show that some populations are opposed to others in relation to some characteristics of pods and/or seeds. In the case of the weakest values (pods and seeds), the populations come from the regions of high altitude and/or with small rainfall; and on the contrary, populations with large seeds and pods come from low regions and/ or with high rainfall (Senhandri Maamri et al.,
2000). Therefore, the morphological approach continues to be the initial step for the classification of many plants: for example, the olive (Rotondi et al., 2003), the cotton (Campbell et al., 2009), wheat (Pagnotta et al., 2009). Lahoz et al. (2011), showed that the cluster analysis is an efficient method for grouping cultivated cultivars, facilitating effective management. Indeed, the analysed Tunisian Retama populations fell into different clusters according to the characterization method used. In conclusion, this paper represents the first study of the genetic diversity of Tunisian Retama populations. This genetic diversity will be furthermore highlighted using molecular markers, although, the morphological descriptors of Retama must be completed by a molecular analysis using RAPD, SSR, or AFLP to understand the genetic organisation of this species in Tunisia.

\section{Salinity and drought treatments}

Salinity stress can affect seed germination through osmotic effects (Welbaum et al., 1990) or by ion toxicity (Huang and Reddman, 1995). In this context, the term "salt stress" during seed germination is used only to refer to situations where the seeds germinate rapidly under salt stress conditions. No distinction is made between osmotic and ionic effects of salinity stress (Bayuelo-Jiménez et al., 2002). Our study indicated that the effect of salt stress on germination was weakly pronounced in the case of the castor bean for the four Retama populations studied and compared to control. The highest germination of Retama raetam was obtained in distilled water. Increasing the concentration of $\mathrm{NaCl}$ significantly reduced the germination percentage. The results obtained in the present experiments corroborate several other studies (Jaouadi et al., 2010; Makhlouf et al., 2015). These results are consistent with those obtained in such other species as Reaumuria vermiculata (Gorai \& Neffati, 2007), Diplotaxis harra (Tlig et al., 2008). In Tunisia, salinity affects large areas mainly in central and south regions where the arid climate increases the proliferation of these territories. The effect of salinity on germination may be explained by osmotic 
and/or toxic effects (Song et al., 2005; Tlig et al., 2008). Seeds of some species are reported to tolerate high salinity during the period when they are dormant in the soil and subsequently germinate when soil salinities are reduced (Khan, Ungar, 1997). Our data showed that seeds of Retama reatam behaved in two characteristic ways according to salinity. First, germination was reduced, indicating that germination was inhibited by salt. Second, seeds showed the phenomenon of "salt stimulation". Retama reatam seeds have the ability to tolerate moderate salinity and osmotic potential, faster and higher germination was recorded. This can be attributed to both ionic and osmotic effects (Song et al., 2005; Gorai, Neffati, 2007; Tlig et al., 2008). High $\mathrm{NaCl}$ significantly affected germination patterns. Although higher salinity generally decreases germination, the detrimental effect of salinity is less severe at the optimum germination osmotic potential. Salt stress decreased both the rate and the percentage of germination of Retama reatam. This result corroborates several other studies revealing that halophytes, as glycophytes, are sensitive to salt during the germination stage (Ungar, 1995; Katembe et al., 1998; Khan et al., 2002; Gorai, Neffati, 2007; Gorai et al., 2011). In saline and dry soils, water potential is not very different to that of desiccated seeds. Therefore, at low osmotic potentials water does not enter the seeds and induce germination. Desert shrubs vary in their ability to germinate in the presence of moisture stress. In our experiments, it should be noted that moisture stress was tested at the most suitable temperature found $\left(25^{\circ} \mathrm{C}\right)$. Results showed that provenance 2 had a higher germination percentage of the stressed seeds at $-0.03 \mathrm{MPa}$ compared with non-stressed seeds. Seed germination percentage and Kotowski's coefficient generally decrease as soil water potential decreases (Evans, Etherington, 1990; Oberbauer, Miller, 1982), either by drought or by higher salinity. By increasing water stress, similar results were found on germination of Diospyros texana seeds that decreased from about $95 \%$ to $45 \%$ at 0 and $-0.6 \mathrm{MPa}$, respectively (Everitt, 1984), whereas germination of three decidu- ous semi-shrubs of genus Artemisia was inhibited severely in $\mathrm{PEG}_{6000}$ solutions at $-1.2 \mathrm{MPa}$ (Tobe et al., 2006). Seed imbibition rate, germination percentage and germination rate generally decrease as soil water potential decreases (Song et al., 2005), either by drought or by higher salinity. Our data show that an increase in osmolality of PEG ${ }_{6000}$ solutions results in decreasing both the percentage and the rate of germination in Retama populations, indicating that water stress inhibits germination. At low osmotic potentials, water does not induce germination. This was in agreement with the germination behaviour of most species (Tobe et al., 2006; Gorai et al., 2009; Maraghni et al., 2010). From the present study it can be concluded that Retama populations have the ability to tolerate salt stress and recover after exposure to $\mathrm{NaCl}$ solutions and osmotic potential concentrations. Further investigations are necessary to understand the early establishment of this species under field conditions and to determine if there are differences between the seed germination stage and early seedling growth in their responses to salinity and drought stresses. In our study, we showed a strong correlation between the seed weight and the germination percentage of Retama populations under salt stress and water stress. In this direction, Ndiaya et al., 2014, find that the most important for germination are seeds of large caliber, while small and medium sizes have low percentages of germination, therefore saline inhibition concerns germination of seeds of small and medium sizes more. Salinity inhibits seed germination of Gossypium hirsutum and the seed size improves the germination ability facing salt stress. It would be more useful to use the larger caliber of seeds in a saltwater environment (Ndiaya et al., 2014).

\section{CONCLUSIONS}

This work was performed as part of the evaluation of the behaviour of Retama raetam populations under water and salt stress in the germination stage. The morphological parameters were measured to identify a relationship between the size of the seeds and their germination ca- 
pacity under salinity and osmotic stress. The results showed that the seed weight is the major factor in promoting germination under stress and confirm that saline water and sprouting inhibition have impact on the smaller caliber of seeds. Also, the results of our study show that salt and osmotic stress affected seed germination of Retama raetam populations by decreasing the germination percentage and the average time for germination and Kotowski's coefficient.

\section{ACKNOWLEDGEMENTS}

We thank Sabri Wellani and all members of CRDA of Nabeul for crucial help and for providing technical assistance. We are grateful to the members of the staff of the Laboratory of Forest Ecology.

\section{Conflict of interest}

The authors declare that they have no conflict of interest.

Received 14 October 2016 Accepted 22 May 2017

\section{References}

1. Abdel Halim OB, Abdel Fattah H, Halim AF, Murakoshi I. Comparative chemical and biological studies of the alkaloid al content of Lygos species and varieties growing in Egypt. Acta Pharm Hung. 1997; 67(6): 241-7.

2. Algandaby MM, Alghamdi HA, Ashour OM, Abdel-Naim AB, Ghareib SA, Abdel-Sattar EA, Hajar AS. Mechanisms of the antihyper glycemic activity of Retama raetam in streptozotocin-induced diabetic rats. Food and Chemical Toxicology. 2010; 48: 2448-753.

3. AL-Tubuly RA, Auzi AA, AL-Etri-Endi AA, Nahar L, Sarker SD. Effects of Retama raetam on the central nervous system in experimental animals. Arch Biol Sci, Belgrade. 2011; 63(4): 1015-1021. doi:10.2298/ABS1104015A.

4. Archer S, Pyke DA. Plant animal interactions affecting establishment and persistence on re- vegetated rangelands. Journal of Range Management. 1991; 44: 558-65.

5. Arzani HM, Zohdi E, Fish GB, Amiri A, Nikkhah DW. Phenological effects on forage quality of five grasses. Journal of Range Management. 2004; 57: 624-9.

6. Assogbadjo AE, Sinsin B, Patrick Vandamme P. Morphological characters and production of baobab tree capsules (Adansonia digitata L.) in Benin. Fruits. 2005; 60: 327-40. doi: 10.1051/fruits:2005039.

7. Baba Aissa F. Encyclopédie des plantes utiles. Flore d'Algérie et du Maghreb. Alger: Librairie Moderne; 1999.

8. Bayuelo-Jiménez JS, Debouck DG, Lynch JP. Salinity tolerance of Phaseolus species during germination and early seedling growth. Crop Science. 2002; 42: 1584-94.

9. Bokhari TBH, Faugeron C, Hachem K, KaidHarche M, Gloaguen V. Investigation of parietal polysaccharides from Retama raetam roots. African Journal of Biotechnology. 2015; 14(29): 2327-34.

10. Boulila F, Depret G, Boulila A, Belhadi D, Benallaoua S, Laguerre G. Retama species growing in different ecological climatic areas of north eastern Algeria have a narrow range of Rhizobia that form a novel phylogenetic clade within the Brady rhizobium genus. Syst Appl Microbiol. 2009; 32: 245-55.

11. Boulos L. Flora of Egypt, Azollacea-Oxalidaceae. Cairo: Al-Hadara Publishing; 1999; 1: 258-9.

12. Caceres A, Giron LM, Martinez AM. Diuretic activity of plants used for the treatments of urinary aliments in Guatemaela. Journal of Ethnopharmacology. 1987; 43: 197-201.

13. Campbell BT, Williams VE, Park W. Using molecular markers and weld performance data to characterize the Pee Dee cotton germplasm resources. Euphytica. 2009; 169: 285-301.

14. Caravaca F, Alguacil MM, Figueroa D, Barea JM, Roldan A. Re-establishment of Retama sphaerocarpa as a target species for reclamation of soil physical and biological properties 
in a semi-arid Mediterranean area. Forest Ecol Manag. 2003; 182: 49-58.

15. Cheriti A, Talhi MF, Belboukhari N, Taleb S, Roussel C. Removal of copper from aqueous solution by Retama raetam Forssk. growing in Algerian Sahara. Desalination and Water Treatment. 2009; 10(1-3): 317-20. http:// dx.doi.org/10.5004/dwt.2009.816.

16. Cooper CE, Withers PC, Mawson PR, Johnstone R, Kirkby T, Prince J, Bradshaw SD, Robertson H. Characteristics of Marri (Corymbia calophylla) fruits in relation to the foraging behaviour of the Forest Red-tailed Black Cockatoo (Calyptorhynchus banksii naso). Journal of the Royal Society of Western Australia. 2003; 86: $139-42$.

17. Dart P. Nitrogen fixation by tropical trees and shrubs. In. Elmerich C, editor: Biological nitrogen fixation for the 21 st century. The Netherlands: Kluwer Academic Press; 1998.

18. Diallo BO, Joly HI, Mckey D, Hossaert-Mckey $\mathrm{M}$, Chevallier $\mathrm{MH}$. Change in biometric characters of seeds and seedlings of nine provenances of Tamarindus indica L. (Caesalpinioideae). Fruits. 2010; 65: 153-67. doi: 10.1051/ fruits/2010010.

19. Eddouks M, Maghrani M, Louedec L, Halouji MJ, Michel B. Antihypertensive activity of the aqueous extract of Retama raetam Forssk. leaves in spontaneously hypertensive rats. Journal of Herbal Pharmacotherapy. 2007; 7: 65-77.

20. Edziri H, Mastouri M, Cheraif I, Aouni M. Chemical composition and antibacterial, antifungal and antioxidant activities of the flower oil of Retama raetam (Forssk.) Webb from Tunisia. Natural Products Research. 2010; 24: 789-96.

21. El Beyrouthy M, Arnold N, Delelis-Dusollier N, Dupont F. Plants used as remedies antirheumatic and antineuralgic in the traditional medicine of Lebanon. J Ethnopharm. 2008; 120: 315-34.

22. El Hamdani N, Fdil R. Evaluation of fatty acids profile and mineral content of Retama monosperma (L.) Boiss. of Morocco. J Mater Environ Sci. 2015; 6(2): 538-45.
23. El-Hilaly JH, Mammouchi M, Lyoussi B. Ethnobotanical studies and economic evaluation of medicinal plants in Taounate province (Northern Morocco). J Ethnopharm. 2003; 86: 149-58.

24. El-Shaer HM. Rangelands as feed resources in the Egyptian desert: Management and improvement. In: Proceedings of the International Conference on Desert Development in the Arab Gulf Countries, State of Kuwait, Kuwait City, 2000.

25. Evans C, Etherington JR. The effect of soil water potential on seed germination of some British plants. New Phytologist. 1990; 115: 539-48.

26. Everitt BS. Introduction to Latent Variable Models. London: Chapman and Hall; 1984. 107 p.

27. Gabriele SA, Kevin S, Goul B, Murray G. Seed biology of Metrosideros excelsa (Myrtaceae), New Zealand Journal of Botany. 2002; 40(3): 419-25, doi:10.1080/0028825X.2002.9512803.

28. Gorai M, Gasmi H, Neffati M. Factors influencing seed germination of medicinal plant Salvia aegyptiaca L. (Lamiaceae). Saudi Journal of Biological Sciences. 2011; 18: 255-60.

29. Gorai M, Neffati M. Germination responses of Reaumuria vermiculata to salinity and temperature. Annals of Applied Biology. 2007; 151: 53-9.

30. Gorai M, Tlig T, Neffati M. Influence of water stress on seed germination characteristics in invasive Diplotaxis harra (Forssk.) Boiss (Brassicaceae) in arid zone of Tunisia. Journal of Phytology. 2009; 1: 249-54.

31. GRIN, Database. 2011 USDA, ARS, National Genetic Re-sources Program. Germplasm Resources Information Network - (GRIN). Online Database. National Germplasm Resources Laboratory, Beltsville, Maryland. URL: http: // www.ars-grin.gov/cgi-bin/npgs/html/taxon. pl?30932.

32. Gutterman Y. Seed germination in desert plants. Berlin: Springer-Verlag; 1993. p. 253.

33. Hayet E, Maha M, Samia A, Mata M, Gros P, Raida H, Ali MM, Mohamed AS, Gutmann L, 
Mighri Z, Mahjoub A. Antimicrobial, antioxidant, and antiviral activities of Retama raetam (Forssk.) Webb flowers growing in Tunisia. World Journal of Microbiology and Biotechnology. 2008; 24: 2933-40.

34. Hayet E, Samia A, Patrick G, Ali M, Maha M, Laurent G, Mighri Z. Mahjoub L. Antimicrobial and cytotoxic activity of Marrubium alysson and Retama raetam grown in Tunisia. Pakistan Journal of Biological Sciences. 2007; 10: 1759-62.

35. Huang J, Reddman RE. Salt tolerance of Hordeum and Brassica species during germination and early growth. Canadian Journal of Plant Science. 1995; 75: 815-9.

36. Izhaki I, Ne'eman G. Hares (Lepu sspp.) as seed dispersers of Retama raetam (Fabaceae) in a sandy landscape. J Arid Environ. 1997; 37: $343-54$.

37. Jaouadi W, Hamrouni L, Souayeh N, Khouja ML. Étude de la germination des graines d'Acacia tortilis sous différentes contraintes abiotiques. Biotechnologie Agronomie Société Environnement. 2010; 14(4): 643-52.

38. Katembe WJ, Ungar IA, Mitchell JP. Effect of Salinity on Germination and Seedling Growth of Two Atriplex species. Annals of Botany. 1998; 82: 167-72.

39. Khan MA, Gul B, Weber DJ. Seed germination in relation to salinity and temperature in Sarcobatus vermiculatus. Biologia Plantarum. 2002; 45: 133-5.

40. Khan MA, Ungar IA. Seed germination and dormancy of Polygonum aviculare L. as influenced by salinity, temperature, and gibberellic acid. Seed Science and Technology. 1997; 26: 107-17.

41. Kigel J. Seed germination in arid and semiarid regions. In: Kigel J, Galili G, editors. Seed development and germination. New York: Marcel Dekker: 1995. p. 645-99.

42. Koriem KMM, Farraqm ARH, Badawy AA, El-Toumy SA. Role of some Egyptian medicinal plants against liver and kidney toxicity induced by cadmium chloride. Toxicological Mechanisms and Methods. 2009; 19: 524-34.
43. Kouyaté AM, Decaluwé E, Guinda F, Diawara H, Diarra I, N'diaye I, Vandamme P. Morphological variability of the baobab tree (Adansonia digitata L.) in Mali. Fruits. 2011; 66: 247-55. doi: 10.1051/fruits/2011032.

44. Kouyaté AM, Van Damme P. Morphological characters of Detarium icrocarpum Guill. and Perr. in southern Mali. Fruits. 2002; 57: 231-8. doi: 10.1051/fruits:2002020.

45. Lahoz I, Fernandez JA, Migliaro D, Macua JI, Egea-Gilabert C. Using molecular markers, nutritional traits and field performance data to characterize cultivated cardoon germplasm resources. Sci Hortic. 2011; 127: 188-97.

46. Laudadio V, Dario M, Hammadi M, Tufarelli V. Nutritional composition of three fodder species browsed by camels (Camelus dromedarius) on arid area of Tunisia. Tropical Animal Health and Production. 2009; 41: 1219-24.

47. Lopez-Pintor A, Espigares T, Rey-Benayas JM, Gomez-Sal A. Effect of simulated parentcreated micro environmental conditions on germination of Retama sphaerocarpa L. Boiss. Seeds. Journal of Mediterranean Ecology. 2000; 1: 219-26.

48. Maghrani M, Lemhadri A, Jouad H, Eddouks M. Effect of the desert plant Retama raetam on glycaemia in normal and streptozotocin-induced diabetic rats. Journal of Ethnopharmacology. 2003; 87: 21-5.

49. Maghrani M, Zeggwagh NA, Haloui M, Eddouks M. Acute diuretic effect of aqueous extract of Retama raetam in normal rats. J Ethnopharm. 2005; 99: 31-5.

50. Mahnane W. Appréciation de la diversité génétique du genre Rétama par les marqueurs biochimiques. Mémoire de magistère, 2009.

51. Makhlouf K, Hamrouni L, Khouja ML, HananamM. Salinity effects on germination, growth and mineral nutrition of Ricinus communis seedlings. Acta Botanica Hungarica. 2015; 57(3-4): 383-400. doi: 10.1556/034.57.2015.3-4.8.

52. Maraghni M, Gorai M, Neffati M. Seed germination at different temperatures and water 
stress levels, and seedling emergence from different depths of Ziziphus lotus. South African Journal of Botany. 2010; 76: 453-9.

53. Mittler R, Merquiol E, Hallak-Herr E, Rachmilevitch S, Kaplan A, Cohen M. Living under a "dormant" canopy: a molecular acclimation mechanism of the desert plant Retama raetam. Plant Journal. 2001; 25: 407-16.

54. Moro MJ, Pugnaire FI, Haase P, Puig de Fabregas J. Effect of the canopy of Retama sphaerocarpaon its under storey in a semiarid environment. Functional Ecology. 1997; 11: 425-31.

55. Naser AM, Barakat VL, Eugenio C, Vincenzo T. Potential Contribution of Retama raetam (Forssk.) Webb \& Berthel as a Forage Shrub in Sinai, Egypt. Arid Land Research and Management. 2013; 27: 257-271. doi: $10.1080 / 15324982.2012 .756561$.

56. Ndiaye M., Ganry F. Variation in the biological $\mathrm{N}_{2}$ fixation by tree legumes in three ecological zones from the north to the south of Senegal. Arid Soil Research Rehabilitation. 1997; 11: 245-54.

57. Niadye A, Faye E, Abdoul Touré M. Effets du stress salin sur la germination des graines de Gossypium hirsutum L. Journal of Applied Biosciences. 2014; 80: 7081-92.

58. Oberbauer S, Miller PC. Effect of water potential on seed germination. Holarctic Ecology. 1982; 5: 218-20.

59. Pagnotta MA, Mondini L, Codianni P, Fares C. Agro-nomical, quality, and molecular characterization of twenty Italian emmer wheat (Triticum dicoccon) accessions. Genet Resour Crop Evol. 2009; 56: 299-310.

60. Quezel P, Santa S. Nouvelle flore de l'Algérie et des régions désertiques méridionales, édition du centre national de la recherche scientifique, 1962.

61. Rotondi A, Magli M, Ricciolini C, Baldoni L. Morphological and molecular analyses for the characterization of a group of Italian olive cultivars. Euphytica. 2003; 132: 129-37.

62. Saada M, Falleh H, Jalleli I, Snoussi M, Ksouri R. Phenolic profile, biological activities and fraction analysis of the medicinal halophyte Retama raetam. South African Journal of Botany. 2014; 94: 114-21. http://dx.doi. org/10.1016/j.sajb.2014.06.010.

63. Seglie L, Scariot V, Larcher F, Devecchi M, Chiavazza PM. In vitro seed germination and seedling propagation in Campanula spp. Plant Biosyst. 2012; 146(1): 15-23.

64. Selami N, Auriac MC, Catrice O, Capela D, KaidHarche M, Timmers T. Morphology and anatomy of root nodules of Retama monosperma (L.) Boiss. Plant Soil. 2014; 379(1-2): 109-119.

65. Senhadri Maamri F, Rahal Bouziane H, Rahmani DH, Abdelguerfi A. Etude biométrique des gousses et des graines de populations de deux générations de Medicago truncatu la Gaert. In: Sulas L. (ed.). Legumes for Mediterranean forage crops, pastures and alternative uses Zaragoza: CIHEAM. Cahiers Options Méditerranéennes. 2000; 45: 249-53.

66. Song J, Feng G, Tian C, Zhang F. Strategies for adaptation of Suaeda physophora, Haloxylon ammodendron and Haloxylon persicumto a saline environment during seed germination stage. Annals of Botany. 2005; 96: 399-405.

67. Tlig T, Gorai M, Neffati M. Germination responses of Diplotaxis harra to temperature and salinity. Flora. 2008; 203: 421-8.

68. Tobe K, Li XM, Omasa K. Effects of sodium chloride on seed, germination and growth of two Chinese desert shrubs, Haloxylon ammodendron and $H$. persicum (Chenopodiaceae). Australian Journal of Botany. 2006; 48: 455-60.

69. Ungar IA. Seed germination and seed-bank ecology of halophytes. In: Kigel J, Galili G (eds.). Seed Development and Germination. Marcel Dekker, New York, 1995; pp. 599-627.

70. Welbaum GE, Tissaoui T, Bradford KJ. Water relations of seed germination in muskmelon $(\mathrm{Cu}$ cumismelo L.): III. Sensitivity of germination to water potential and abscisic acid during development. Plant Physiology. 1990; 92: 1029-37.

71. Youssef AM. Seed germination of some desert plants from Egypt. J Appl Sci Res. 2009; 5(2): 144-50. 
Kaouther Mechergui, Hela Mahmoudi, Mohamed Larbi Khouja, Wahbi Jaouadi

VEIKSNIAI, TURINTYS İTAKOS PAŠARINIŲ

AUGALŲ (RETAMA RAETAM SUBSP. BOVEI (FABACEAE) SĖKLŲ DAIGUMUI: INTERAKTYVUS POVEIKIS VAISIU MORFOLOGIJAI DRUSKINGUMO IR OSMOSINIO STRESO SĄLYGOMIS

\section{Santrauka}

Retama gentis priklauso pupinių (Fabaceae) šeimai. Retama raetam rūšies augalai daugiausia auga kopose ir dykumose; čia jie pakenčia ypač didelę sausrą, todèl dažnai yra naudojami kopoms stabilizuoti ir dykumoms atkurti. Šio tyrimo metu buvo analizuojama keturių augalo Retama raetam populiacijų sèklų morfologija, druskingumo ir osmosinio streso poveikis daigumui Tunise. Sèklos buvo surinktos iš
Bouhedma nacionalinio parko Meknassi, Oueslatia ir Rtiba populiacijų. Rezultatai rodo, kad ši rūšis gali pakęsti iki $15 \mathrm{~g} / \mathrm{l}$ druskingumą (Rtiba populiacijos daigumas siekè $42 \%$ ) ir toleruoti didelę sausrą $\mathrm{PEG}_{6000}$ (Oueslatia populiacijos daigumas siekè $29 \%$ esant -1,6 MPa vandens potencialui). Buvo išmatuotos sèklų morfologinès savybès siekiant nustatyti ryšį tarp sẻklų dydžio ir jų daigumo druskingumo ir osmosinio streso sąlygomis. Rezultatai atskleidè koreliaciją tarp sẻklų morfologinių savybių ir daigumo procento. Sèklų svoris yra pagrindinis daigumą skatinantis veiksnys streso metu, taip pat patvirtina, kad sèklų dydis priklauso nuo sūraus vandens ir ūglių augimo slopinimo.

Raktažodžiai: Retama raetam, populiacija, morfologinès savybès, druskingumo stresas, osmosinis stresas 